\title{
Linx
}

Revue des linguistes de l'université Paris X Nanterre

47 | 2002

Du sens au sens

\section{Des relatives de Toussaint. Une syntaxe du regard}

On the relatives clauses in a novel by Toussaint. Syntax and perception

\section{Rémy Porquier}

\section{OpenEdition}

Journals

Édition électronique

URL : http://journals.openedition.org/linx/589

DOI : $10.4000 /$ linx.589

ISSN : 2118-9692

\section{Éditeur}

Presses universitaires de Paris Nanterre

\section{Édition imprimée}

Date de publication : 1 décembre 2002

Pagination : 121-132

ISSN : 0246-8743

\section{Référence électronique}

Rémy Porquier, « Des relatives de Toussaint. Une syntaxe du regard », Linx [En ligne], 47 | 2002, mis en ligne le 01 juin 2003, consulté le 06 mai 2019. URL : http://journals.openedition.org/linx/589 ; DOI : 10.4000/linx.589 


\title{
Des relatives de Toussaint. Une syntaxe du regard
}

\author{
Rémy Porquier, Paris $X$-Nanterre, Modyco, UMR 7114 Paris \\ $X / C N R S$
}

De longue date, les écrivains fournissent aux grammairiens un vivier abondant pour illustrer et exemplifier des emplois de structures ou de tournures grammaticales des plus diverses. Il n'est pas rare par ailleurs de décrire, de caractériser et d'expliquer le style d'un(e) auteur(e) (ou d'une école) en termes d'écart ou de singularité, par la façon dont il/elle utilise de façon originale et/ou récurrente telles ressources grammaticales de la langue dans laquelle elle(il) écrit. Dans les deux cas, il existe de grands fournisseurs d'exemples aux grammaires, certains même parfois restés plus illustres à ce titre que par leur notoriété littéraire. Mais bien d'autres exemples proviennent de sources non littéraires, ou de textes littéraires moins notoires, voire inaperçus.

Je restai encore un instant sur le bord de la route à regarder la maison, et je m'apprêtais à rentrer à l'hôtel quand je remarquai la présence d'une boîte aux lettres sur la grille de la porte d'entrée, qui pendait dans l'obscurité à mi-hauteur d'homme environ, bancale, fixée à un barreau par un fil de fer tordu.

Cette phrase est extraite (page 18) de La réticence, roman de Jean-Philippe Toussaint, paru en $1991^{1}$. Elle illustre un type de proposition relative souvent signalé par les grammaires, sous l'appellation de relatives "détachées », "décrochées » ou « différées».

Bien d'autres passages (voir en Annexe), n'était leur longueur, fourniraient des exemples de choix pour le chapitre « relatives » des grammaires. Mais on s'intéressera particulièrement aux relatives détachées et aux relatives déictiques. On s'arrêtera

${ }^{1}$ Paris, Editions de Minuit, 1991. J.P. Toussaint est également l'auteur de La salle de bain (1985), Monsieur (1986), L'appareil-photo (1989), La télévision (1997), Autoportrait (à l'étranger) (2000), Faire l'amour (2002). 
Rémy Porquier

d'abord sur ce qu'en disent les grammaires et les ouvrages ou articles consacrés aux relatives en français.

\section{Ce que disent les grammaires}

Les grammaires du français moderne et contemporain et les descriptions les plus récentes du français s'accordent à distinguer divers types de relatives, selon leurs caractéristiques syntaxiques et leurs fonctions. Ainsi distingue-t-on les relatives déterminatives (ou restrictives) des relatives explicatives (ou appositives) : celles-ci introduisent un développement, dont la suppression ne change pas le sens de la phrase, et qui équivaut à une coordination ou à une subordination ; celles-là caractérisent l'antécédent à la manière d'un adjectif, et ne peuvent être supprimées sans changer le sens de la phrase :

Le pronom [relatif] introduit une proposition qui qualifie l'antécédent à la façon d'un adjectif [...]. La proposition est subordonnée à l'antécédent comme le serait une épithète $[\ldots .$.$] : L'homme que Kyo cherchait était présent. Une telle proposition est dite$ « déterminative ».

Le pronom introduit une proposition qui est jointe à la principale par un lien parfois proche de la coordination : A ce moment, un paysan âgé se présenta à la porte avec une brassée de bois qu'il jeta à la porte. Une telle proposition est dite " explicative».

(Grammaire Larousse du français contemporain)

Cette distinction est souvent établie et formulée en terme d'« extension $»^{2}$ (voir Grevisse 1993) :

On distingue d'après le sens :

- la relative déterminative, qui restreint l'extension [...] du terme qu'elle accompagne (la suppression de la relative modifierait profondément le message) : Le Président de la République qui est mort en 1974 est Georges Pompidou [...] et ne doit pas être séparée de l'antécédent par une virgule

- la relative explicative, qui ne restreint pas l'extension du terme qu'elle accompagne (la suppression de la relative ne modifierait pas profondément le message) : Le Président de la République, qui est le chef des armées, préside les conseils supérieurs de la Défense Nationale [et] est séparée de l'antécédent par une virgule.

(Nouvelle grammaire française)

La distinction entre ces deux types de relatives a fait et fait encore l'objet de nombreux débats, touchant soit à la pertinence de cette distinction, selon le point de vue adopté, soit à la nature de la distinction et donc à la manière de la décrire et/ou de l'expliquer.

2 On notera, dans la citation qui suit, l'emploi méta-référentiel des relatives («qui restreint l'extension du terme qu'elle accompagne »), où des relatives explicatives et déterminatives servent à expliquer aussi bien l'emploi de celles-ci que de celles-là. On en trouvera certainement d'autres exemples, aussi bien que dans toute grammaire, dans le présent article. 
Un autre type de relatives est constitué par celles dénommées, selon les linguistes et les grammairiens qui en traitent, "prédicatives », « déictiques », "attributives » ou encore « indépendantes».

On les trouve après les verbes de perception (Je vois Pierre qui se promène). On les trouve aussi avec les verbes être, rester, se trouver, suivis d'une détermination de lieu (il est là qui t'appelle), avec les présentatifs voici, voilà, c'est, il y a (voilà Pierre qui pleure)

(Grammaire d'aujourd'bui)

[les propositions relatives attributives] déterminent leur antécédent par l'intermédiaire d'un verbe (verbes de perception : voir, entendre, etc. ; verbes trouver, montrer) qui a cet antécédent pour complément d'objet [...] : Je l'aperçois qui vient (Corneille) [...] J'entends mon matelot qui fredonne (V. Hugo)

(Grammaire du français classique et moderne)

On trouve comme complément d'objet des verbes voir, regarder, entendre, écouter, sentir des relatives introduites par qui, qui constituent le propos de la phrase : ce sont des relatives prédicatives (ou attributives): J'ai entendu un oiseau qui chantait - je l'ai entendu qui chantait.

On peut également trouver une relative de ce type après les présentatifs voici et ily $a$ :

Voici le train qui arrive/Le voici qui arrive [...]

(Grammaire méthodique du français)

Quant aux critères formels permettant de distinguer ou de caractériser différentiellement ces trois types de relatives, car il s'agit bien de trois types disjoints, on notera brièvement ici qu'ils ne peuvent se réduire à la présence/absence de la virgule. Si, à l'écrit, celle-ci sert ou aide souvent à distinguer les déterminatives (absence de virgule) des appositives, ce critère n'a pas de pertinence pour les relatives déictiques ni dans le cas d'incises ni lorsqu'on a affaire à des relatives « décrochées ».

\section{Les relatives décrochées ou différées}

Du point de vue syntaxique, il est spécifié ou implicitement admis que le pronom relatif suit immédiatement son "antécédent » ${ }^{3}$, celui-ci étant en principe, ou le plus souvent, un groupe nominal. Cela ne vaut pas dans le cas des relatives déictiques lorsque l'antécédent est pronominalisé, comme dans Le voilà qui arrive, ou absent comme dans Voilà qui m'étonne.

Cependant, la plupart des grammaires du français font mention de relatives dites selon les auteurs séparées, détachées, décrochées, ou encore extraposées (Godard) ou différées (Tesnière), où le pronom relatif se trouve séparé, de quelque manière, de son

\footnotetext{
${ }^{3}$ On voit bien ici l'ambiguïté, instaurée par l'usage, de tels termes: si «antécédent» signifie "précédant dans la phrase», cela ne signifie pas «immédiatement précédent»; le terme « antécédent » n'est d'ailleurs le plus souvent pas compris ainsi mais plutôt comme «ce à quoi (se) relie le relatif », comme le montrent les formulations les plus classiques de la règle d'accord du participe passé.
} 
Rémy Porquier

antécédent, ou dans lesquelles se trouve interposé un élément quelconque entre le pronom relatif et ce à quoi il réfère.

L'usage veut que la proposition relative suive directement l'antécédent du relatif, que celui-ci soit un terme simple ou un groupe (substantif + adjectif ou complément déterminatif) [...] Dans la langue écrite littéraire, la relative peut toutefois être séparée de son antécédent par un terme ou un groupe : Alors une vague vint la chercher, qui s'était toujours tenue à quelque distance du village dans une visible réserve.(Supervielle)

(Grammaire du français classique et moderne)

La règle de proximité immédiate du relatif avec son antécédent, qui a souvent pour effet d'interrompre une proposition, évite d'éventuelles ambiguïtés.

(Grammaire d'aujourd'bui)

Il faut éviter, pour la clarté de la phrase, que la relative soit séparée de l'antécédent par un autre nom : *je signalerai un chapitre dans ce livre qui me paraît beau [...] Dans la langue écrite surtout, la relative se rapportant au sujet est parfois rattachée au prédicat [...] : Une servante entra qui apportait la lampe (Gide).

(Nouvelle grammaire française)

Le pronom relatif suit directement l'antécédent, pour éviter toute équivoque [...] Cependant, et surtout quand il introduit une proposition explicative, il jouit parfois d'une plus grande liberté. Il peut être séparé de l’antécédent : par un pronom apposé [...], par un verbe dont le complément d'objet est l'antécédent (Elle l'entendit qui demandait à Marie de Laclos s'il s'était servi. Mauriac); par un verbe ou un ensemble verbal (La partie est perdue que je ne pouvais gagner qu'avec elle. Gide)

(Grammaire Larousse du français contemporain)

Les observations sur ces relatives décrochées, souvent agrémentées de considérations normatives, signalent, dans des exemples parfois pittoresques ${ }^{4}$, les ambiguïtés qu'elles occasionnent, ou les identifient comme relevant d'un style soutenu ou recherché, illustré par des exemples d'écrivains ( Il la trouvait mignonne, et belle, et délicate / Qui miaulait d'un ton fort doux. La Fontaine).

On notera ici que ce type de relatives se rencontre dans certaines formes d'oral soutenu, comme dans des interviews radiophoniques ${ }^{5}$ :

Tous les Français le savent qui nous écoutent (Giscard d'Estaing, 25 mars 1993, 8h40, France-Inter).

J'ai fait en sorte que ce petit livre soit très peu cher qui contient l'ensemble des éléments [...]

4 «Dans le style relâché, il s'agit généralement d’une gaucherie qui entraîne souvent des quiproquos réjouissants sur la personne de l'antécédent. Elle a acheté un caleçon à son mari, qu'elle a payé très cher. (Entendu par l'auteur, LT, vers 1944). Les almanachs humoristiques foisonnent d'exemples comme : J'ai offert un ravissant petit chat à ma femme, qui a les oreilles noires.» (Tesnière, 1965, 578). Il est curieux de trouver chez divers auteurs des exemples voisins, concernant des oreilles de chats ou de chiens.

${ }^{5}$ L'absence résolue de virgules dans ces transcriptions [les nôtres] de l'oral illustre dans au moins deux des cas le problème que pose l'écrit en la matière. 
(Arnaud Montebourg, 23 mai 2001, 8h25, France-Inter).

Ca ne changera pas le jugement d'un iota que j'ai sur lui. (Claude Allègre, 5 juin 2001, 20h10, France-Inter).

Tous les pays l'ont compris qui ont des structures infiniment plus petites (X, 13 novembre 2001, $22 \mathrm{~h} 20$, France-Inter).

ou dans des textes de presse :

[...] la symbolique est forte qui permet à l'administration américaine, trois mois après les attentats de New-York et Washington, d'annoncer [...] Libération, 12 décembre 2001, p. 3

La loi Fabius vient de passer, qui multiplie les plans d'épargne salariale (Charlie-Hebdo, 5 décembre 2001, p. 5).

La statistique est édifiante qui mérite d'être élargie au-delà des seules périodes de cohabitation:

[...] (Le Monde, 11 janvier 2002, p. 1).

Le Conseil est clair qui établit une doctrine primordiale, laquelle relie directement l'emploi à la compétitivité de l'entreprise (Le Figaro, cité par Le Monde, 15 janvier 2002, p. 22).

La Coupe de la Ligue tombe à pic, qui offre l'Europe à bon marché (L'Équipe, 25 janvier 2002, p. 6).

\section{Les relatives dans La réticence}

Dans ce roman de Jean-Philippe Toussaint, de la page 18 (huitième page effective du texte) à la page 159 (page finale), on ne trouve pas moins de 111 relatives détachées ou décrochées, donc séparées de leur antécédent si l'on considère celui-ci comme l'élément nominal ou le groupe nominal précédant la relative, celle-ci étant anaphorique de celui-là $\grave{a}^{6}$ Pour préciser l'observation quantitative, il faut ajouter que ce ne sont là qu'une partie des très nombreuses relatives du roman, certaines s'enchaînant et s'imbriquant dans des phrases parfois très longues (voir en Annexe et pp. 15, 23, 27, 51, 98-99, 122, 124, 159).

Cette caractéristique n'a pas été relevée dans les autres romans du même auteur, d'où les relatives décrochées sont absentes ou rares?

Pour certaines, l'ambiguité déjà évoquée (quel est l'antécédent?...) est présente : on ne sait de façon certaine quel est, de l'élément nominal immédiatement précédent, ou d'un autre antérieur, celui qu'anaphorise la relative :

Je regardais la route qui montait vers la maison de Biaggi qui était déserte devant moi, p. 13.

\section{La structure des relatives décrochées}

1. Ces relatives sont, dans leur grande majorité, en qui, sauf quelques-unes :

\footnotetext{
${ }^{6} \mathrm{Il}$ est encore plus rare que cet élément nominal suive le pronom relatif (« Il voit, qui file, un rat»), voir Grevisse, 1993, p. 1588.

${ }^{7}$ Une dans La salle de bain, p. 3 : « Le sol semblait sombre, dont le linoleum se décollait par endroits»; dix dans La télévision (pp. 53, 61, 168, 171, 185, 193, 209, 221, 229, 250, 251, 252).
} 
Rémy Porquier

- en dont: mon fils dormait derrière moi, dont j'entendais le souffle régulier, p. 44, Une minuterie se déclencha aussitôt, dont les pulsations résonnaient, p. 67, aussi pp. 71, 81, 84, 123, 145,

- en que : je remarquai la présence de deux lettres posées sur le bureau, que j’allai ramasser, p. 59 , aussi pp. $61,128,129,132,138$,

- en où : Le bain lui plaisait toujours en général, où il pouvait ainsi faire à chaque fois de nouvelles expériences, p. 108, aussi pp. 60, 125,

- en lesquelles: Les vitres étaient très sombres en face de moi, derrière lesquelles le rideau métallique était baissé, p.120, aussi pp. 145, 148.

2. Pour les relatives en qui, de très loin les plus nombreuses, on observe d'abord la diversité des éléments séparant le pronom relatif de l'antécédent :

- groupe verbal ou syntagme verbal : la voiture tournait aussi, qui continuait de nous suivre, p. 33 ; l'homme n'avait pas bougé de la fenêtre, qui avait allumé une cigarette, p. 150); y compris des groupes ou syntagmes prédicatifs : le cadavre était là, qui flottait dans la pénombre, p. 36 ; les eaux du port étaient parfaitement vides en face de moi, qui s'étendaient en silence dans la nuit, p. 102,

- groupe prépositionnel : je remarquai la présence de deux lettres posées sur le bureau, que j'allai ramasser [...], p. 59 ; je vis apparaître la silhouette du patron dans l'embrasure de la fenêtre, qui portait un vieux maillot de corps [...], p. 65 ; j'aperçus immédiatement mon fils dans la pénombre, qui était couché dans son lit de voyage, p. 67,

- groupe adjectival : l'averse se déclencha, soudaine et brutale, ample et bruyante, qui commença à inonder toutes les allées du parc, p. 145,

- subordonnées : mon fils ne m'entendit pas quand je rentrai dans ma chambre, qui dormait tranquillement dans son lit, p. 125 ; y compris des subordonnées relatives,

- divers inserts : et Biaggi se trouvait là dissimulé dans l'ombre, me semblait-il, qui observait tous mes déplacements, p. 56

Plusieurs de ces éléments peuvent se trouver combinés, parfois dans des séquences relativement longues et/ou complexes, entre antécédent et relatif, y compris des relatives :

- J'apercevais le couloir du premier étage en haut des marches où Biaggi se tenait sans bouger peutêtre, qui était là au premier étage en train de m'observer debout dans l'ombre du couloir, p. 60

- Le taxi entra dans le village que j'aperçus de loin qui venait à notre rencontre, p. 32

- Les deux maisons qui se dressaient dans le tournant étaient fermées et silencieuses, que j’apercevais derrière les parois de verre, p. 132

- l'homme cria très fort alors, qui me fit sursauter, jeta le chiffon qu'il avait à la main dans ma direction, qui alla atterrir mollement sur la jetée, p. 156 
- une jeune femme que je n'avais jamais vue était là dans un canapé, qui feuilletait lentement un vieux magarine, p. 107

- c'était la vieille Mercedes grise dont l'aile avant était accidentée que j'avais apercue la veille dans la propriété des Biaggi, p. 33.

Au vu des occurrences relevées, et de l'extrême diversité des agencements syntaxiques des relatives décrochées et de leurs contextes immédiats, on peut s'interroger sur leur statut: déterminatives ? appositives ? Les quelques exemples cidessus, parmi la bonne centaine recensée dans le roman, montrent que la présence/absence de la virgule n'est pas là un critère pertinent : d'une part, la présence d'une virgule paraît en plusieurs cas être fonction de la longueur ou de la complexité de la phrase ${ }^{8}$; d'autre part, un examen plus attentif du corpus montre la présence d'une proportion importante d'un type spécifique de relatives déictiques, introduites ou orientées par des verbes de perception.

\section{Des relatives déictiques?}

Les relatives décrochées inventoriées ressortissent dans leur majorité à trois grands types, classés ici selon le type de verbe les précédant (il s'agit ici dans tous les cas, pour les relatives en qui, du verbe ayant pour sujet l'antécédent) :

$1^{\circ}$ celles où la relative est précédée d'un verbe prédicatif (être, devenir) + adjectif :

Les eaux du port étaient parfaitement vides en face de moi, qui s'étendaient en silence dans la nuit, p. 102

Le couvercle était ouvert, qui pendait le long de la grille, p. 84

La route devint plus sombre encore devant moi, qui montait vers le hameau, p. 53

$2^{\circ}$ celles où la relative est précédée d'un verbe à valeur spatiale (localisation ou déplacement) le plus souvent suivi d'un adverbe de lieu, d'une préposition ou locution prépositionnelle de lieu :

Elles étaient là, les trois lettres que j'avais restituées ce matin, qui reposaient l'une à côté de l'autre sur le bureau, p. 117

Le taxi entra dans le village que j’aperçus de loin qui venait à notre rencontre, p. 31

Sa femme restait dans la cuisine qui passait parfois la tête [...], p. 40

$3^{\circ}$ celles où la relative est précédée d'un verbe de perception du type voir, regarder:

je regardais la route qui montait vers la maison des Biaggi qui était déserte devant moi, p. 137 j'apercevais le long faisceau lumineux du phare de l'île de Sarsuelo qui filait dans le ciel, p. 119 je voyais sa silhouette en transparence dans la cabine téléphonique, qui avait décroché et qui était en train de composer le numéro, p. 130.

${ }^{8}$ Sans doute faudrait-il, pour en savoir plus, interroger (avoir interrogé alors) l’auteur, et peut-être les relecteurs et les correcteurs du roman. 
Rémy Porquier

Diverses imbrications et combinaisons, dans le fil du texte lui-même, peuvent être observées, selon diverses dépendances, entre ces relatives déictiques et les deux autres types de relatives. On en trouvera en annexe divers exemples.

Ces trois types correspondent bien aux diverses relatives déictiques (ou attributives ou prédicatives) déjà identifiées et distinguées par les grammairiens. Mais le troisième demande un examen plus précis..

L'inventaire des verbes concerne là en premier lieu la perception visuelle (voir, regarder, apercevoir, remarquer, apparaître, voir apparaître, être visible, se dessiner, revoir, ...) mais aussi d'autres modes de perception :

J'entendis quelqu'un marcher à l'étage en-dessous, qui, presque aussitôt, s'engagea [...], p. 78

L'bomme cria très fort alors, qui me fit sursauter, p. 152.

J'entendis le bruit du moteur qui approchait [...], p. 5.

D'autre part, l'attention apportée aux verbes de perception comme verbes « antécédents » à la relative amène, dictature ou persuasion du corpus, à examiner leur présence à l'intérieur même des relatives décrochées en question :

Mon fils dormait derrière moi, dont j'entendais le souffle régulier, p. 81

L'bomme ne bougeait pas à côté de moi, qui regardait dehors lui aussi, p. 151

Les deux maisons qui se dressaient dans le tournant étaient fermées et silencieuses, que j’apercevais derrière les parois de verre [...], p.132.

On remarque alors, à l'intérieur même des relatives,

$1^{\circ}$ que les verbes des relatives sont en partie de même nature que ceux qui précèdent les relatives : verbes prédicatifs, verbes spatiaux et verbes de perception ;

$2^{\circ}$ qu'on y retrouve des verbes de perception analogues, bien que moins fréquents, à ceux précédant les relatives :

Les deux maisons qui se dressaient dans le tournant étaient fermées et silencieuses, que j’apercevais derrière les parois de verre [...], p. 132

Mon fils était réveillé maintenant, que j’entendais gazouiller, p. 138.

Par ailleurs, le sujet de la perception, et de la relative, est le plus souvent le narrateur (« je voyais », « je remarquai ») mais parfois un autre personnage :

L'bomme ne m'avait toujours pas vu, qui continuait de ratisser dans le parc, p. 142

L'bomme m'avait vu maintenant, qui cessa de ratisser quand j'entrai dans la propriété et qui me regarda venir vers lui sans bouger, p. 143

Mon fils ne m'entendit pas quand je rentrai dans ma chambre, qui dormait tranquillement dans son lit, p. 125 ,

même si ce qui est narré, et vu, l'est par le narrateur-énonciateur (= 'Je vis que] l'homme (ne) m'avait (pas) vu').

On peut en conclure que l'ensemble des phrases à relatives décrochées, dans La réticence, est dominé par le schème

(Verbe de perception) + Relatif $+($ Verbe de perception $)$ 
que l'on retrouve par ailleurs dans un nombre important de relatives «non décrochées ».

De façon plus large, la saillance des verbes de perception, d'abord repérée à propos des relatives, est par ailleurs l'un des traits du roman La réticence, irrigué par le vocabulaire de la vision : regarder (100 occurrences), voir (47 occ.), apercevoir (39 occ.), observer, jeter un coup d'ail, suivre d'un cil, relever les yeux, d'un coup d'cil, jeter un regard, remarquer, guetter, examiner, scruter, apparaître, disparaître, observer, se deviner, être visible, image, champ de vision, etc. Plus largement, un pourcentage important de verbes ou de locutions verbales, dans des relatives - différées ou non - ou en dehors de celles-ci, ressortissent à la perception : briller, miroiter, bruissement, sombre, pénombre, etc.

\section{Conclusion}

On a pu constater le lien étroit, dans La réticence, entre les relatives « différées » et les relatives déictiques, particulièrement celles introduites par un verbe de perception. Ce lien s'explique en partie par le caractère narratif-descriptif du roman, où l'auteur-narrateur, du début à la fin, décrit ce qu'il voit et perçoit, ce qui est vu et perçu. La structure des relatives différées, plutôt que de marquer quelque rupture ou une forme de syncope ${ }^{9}$, tente apparemment de reproduire en texte, en le segmentant et en l'ordonnant de façon séquentielle, le flux, le fur et à mesure, le continu rythmé de la perception.

Mais cette singularité, constitutive de l'écriture du roman, révèle ou illustre un autre aspect du fonctionnement des relatives en français. Henry (1975), Le Goffic (1979), Kleiber (1987), Wilmet (1997) et bien d'autres, selon divers points de vue, mettent en question la distinction classique entre relatives déterminatives et appositives. Tesnière, bien auparavant (1965, pp. 313-319 et 557-564), signale et commente la dualité de la proposition relative et la «nature double » - translatif et jonctif, selon sa terminologie - du pronom relatif : hypotaxique et parataxique. De cette dualité, nombre des relatives déictiques différées de La réticence portent la marque, éclairant du même coup celle des autres déictiques et des autres différées, et celle des relatives «en cascade » telles que celles mentionnées en Annexe ou celles de la phrase suivante :

J'étais assis là tout seul en manteau sombre sur la plage, les pieds nus dans le sable mouillé, et je vis un bateau apparaitre à l'horizon alors, un ferry qui glissait lentement devant moi tout illuminé dans la nuit, qui glissait immobile à la surface de l'eau et qui finit par disparaître très lentement derrière l'île de Sasuelo. (p. 159),

qui vient clore le roman.

Rémy PORQUIER

Département des sciences du langage Université Paris X

\footnotetext{
${ }^{9} \mathrm{Ce}$ qui aurait $\mathrm{pu}$, en première hypothèse, justifier ou expliquer le titre du roman. Une autre hypothèse consisterait, dans le paradigme des titres des romans de Toussaint (L'appareil photo, La télévision, Autoportrait); à y lire «La rétine-sens »... Jean-Philippe Toussaint est également auteur et metteur en scène de cinéma: La salle de bain, La sévillane, La patinoire.
} 
92001 Nanterre cedex porquier@u-paris10.fr

\section{BIBLIOGRAPHIE}

ARRIVÉ, M., GADET, F., GALMICHE, M., 1986, La grammaire d'aujourd'bui. Paris, Flammarion.

ARrivÉ, M., BLANCHE-BENVENISTE, C., CHEVAlIER, J.C., PEYTARD, J., 1964, Grammaire Larousse du français contemporain. Paris, Larousse.

BENZAKOUR, F., 1984, «Les relatives déictiques », in Kleiber, G. (dir.) : Recherches en pragmasémantique. Paris, Kincksieck, 75-106.

CADIOT, P., 1976, « Relatives et infinitives « déictiques » en français », DRL AV , 13, 1-64.

DAMOURETTE, J., PICHON, E., 1911-1940, Des mots à la pensée. Essai de grammaire de la langue française. Paris, d'Artrey.

FUCHS, C. (dir.), 1987, « Avant-propos », Langages, 88, 5-7.

GODARD, D., 1988, La syntaxe des relatives en français. Paris, Ed. du CNRS.

Grand Larousse de la langue française, 1977, Paris, Larousse [Tome 6, 5019-5033].

GREVISSE, M., 1993, Le bon usage (13e édition refondue par A. Goosse). Paris-Louvain, Duculot.

GREVISSE, M., GOOSSE, A., 1980, Nouvelle grammaire française. Paris-Gembloux, Duculot.

HENRY, A., 1975, «Constructions relatives et articulations discursives », Langages, 37, 81-98.

KLEIBER, G., 1987, «Relatives restrictives et relatives appositives: dépassement(s) autorisé(s) », Langages, 88, 41-63.

LE GOFFIC, P., 1979, «Propositions relatives, identification et ambiguïté, ou : pour en finir avec les deux types de relatives ", DRL $A V, 21,135-145$.

PÉCHEUX, M., 1990, «Effets discursifs liés au fonctionnement des relatives en français ». Dans L'inquiétude du discours. [Textes choisis et présentés par Denise Maldidier]. Paris, Editions des Cendres, 273-280.

PREBENSEN, H., 1982, «La proposition relative dite attributive », Revue romane, XVII, 98-117.

RIEGEL, M., PELLAT, J.-C., RIOUL, R., 1994, Grammaire méthodique du français. Paris, Presses Universitaires de France.

ROTHENBERG, M., 1971, «Propositions relatives à antécédent explicite introduites par des présentatifs ». Etudes de Linguistique Appliquée, 2, 102-117.

ROTHENBERG, M., 1972, «Les propositions relatives adjointes en français ». Bulletin de la Société de Linguistique, 67, 1, 175-213.

SANDFELD, K., 1936, Syntaxe du français contemporain. Paris, Champion. 
SCHWARZE, C., 1974, «Les constructions du type « je le vois qui arrive » », in C. Rohrer \& N. Ruwet (dirs): Actes du colloque franco-allemand de grammaire transformationnelle. Etudes de syntaxe. Tübingen, Niemeyer, 18-30.

TESNIÈRE, L., 1965, Éléments de syntaxe structurale. Paris, Klincksieck.

TOURATIER, C., 1980, La relative. Essai de théorie syntaxique. Paris, Klincksieck.

VOGELEER, S., 1994, «L'accès perceptuel à l'information : à propos des expressions 'un homme arrive', 'on voit arriver un homme' ", Langue française 102, 69-83.

WAGNER, R.-L., Pinchon, J., 1991, Grammaire du français classique et moderne. Paris, Hachette.

WILMET, M., 1997, Grammaire critique du français. Louvain-Paris, Duculot.

\section{ANNEXE}

Devant la capitainerie du port, un peu plus loin, une grosse barque de pêche était en réfection, qui avait été montée sur cales au milieu de l'aire de carénage, et deux hommes se tenaient là qui avaient l'air de s'entretenir de la coque du bateau, me semblait-il, alors qu'un troisième, assis au volant de sa voiture et la portière ouverte, les regardait discuter en intervenant parfois dans la conversation pour réfuter toutes les suggestions qu'ils pouvaient faire avec une sorte de fatalisme résigné que ses compagnons acceptaient toujours de bonne grâce d'ailleurs, comme si l'homme de la voiture était le patron et qu'il était effectivement foutu, son bateau. (pp. 24-25)

Je ne savais pas s'il avait réussi à résoudre son problème, mais il venait de prendre place à une table juste en face de moi, accompagné de trois dames blondes qui devaient avoir le même coiffeur, toutes trois très convenables et ayant été jeunes, souriantes et amies, qui se prenaient familièrement l'avant-bras par-dessus la table dans un tintement de bracelets et se faisaient expliquer le menu par le patron qu'elles semblaient connaittre de longue date et qu'elles appelaient par son prénom, appelant Georges non seulement le patron d'ailleurs, mais aussi l'homme de la voiture, qui, impassible sur sa chaise derrière ses lunettes à verres teintés, intervenait parfois dans la conversation pour réfuter avec constance toutes les suggestions qui pouvaient lui être faites sur le choix de l'entrée. (p. 27)

Il y avait une petite fontaine au milieu de la place, où la pluie tombait dans un bruissement imperceptible, et trois bancs en mauvais états étaient abandonnés là, qui avaient dû être verts en leur temps et qui étaient presque grisâtres maintenant, tout écaillés, vides tous les trois à l'exception de celui du milieu, où était assis un unique vieux dont je n'avais pas tout de suite remarqué la présence sous la casquette. (p. 31)

Une voiture nous suivait, dont les veilleuses étaient également allumées dans le faible brouillard qui enveloppait la route, et, au dernier embranchement qui menait à Sasuelo, je remarquai que la voiture tournait aussi, qui continuait de nous suivre à distance sous la pluie. (p. 33) 
Je marchais tranquillement sur le bord de la route, et mon fils se tenait devant moi, la tête bien droite dans la poussette comme si je l'avais chargé de quelque mission particulière de vigie à l'avant du convoi, tâche dont il s'acquittait avec le plus grand sérieux d'ailleurs, l'œil aux aguets sous la cagoule - un seul œil, car sa cagoule n'était pas très bien remontée sur son front et lui éborgnait un peu l'autre œil -, à l'affût de tout ce qui pouvait bouger devant lui, fût-ce une simple feuille morte emportée par le vent dont il suivait les pérégrinations d'un œil soucieux depuis le macadam de la chaussée où elle avait commencé à s'envoler jusqu'à son point d'arrivée sur le bord de la route, où quelque touffe d'herbes humides venait l'arrêter. (p. 83)

Car Biaggi se trouvait sur l'île de Sasuelo en réalité, le cadavre de Biaggi raide et déjà décomposé dans son caban mouillé se trouvait sur l'île de Sasuelo en ce moment, qui, après avoir flotté un moment sur le dos dans les eaux noires du port, avait dû être repêché et hissé à bord d'une barque de pêche qui avait pris la mer sous le même clair de lune que celui de cette nuit, le même exactement, avec les mêmes nuages noirs qui glissaient dans le ciel, et, lorsque la barque qui filait lentement dans la nuit en direction de l'île de Sasuelo avait fini par arriver en vue des côtes accidentées de l'île, elle avait accosté doucement le long d'un petit ponton de débarquement qui se dressait dans la pénombre au bord de l'eau, et le cadavre de Biaggi avait alors été débarqué sur le rivage sous la lumière argentée de la lune, le visage bleui et tuméfié violemment éclairé par la lumière du phare dont la haute silhouette silencieuse se dressait là tout près dans la nuit, puis, lentement, le corps avait été traîné dans l'obscurité le long du petit chemin de pierres tracé à même la paroi rocheuse qui montait vers la cabine du phare. (p. 124) 